\title{
Terapia de conversión en cáncer gástrico estadio IV: a propósito de un caso
}

\section{Case Report of Conversion Therapy in Stage IV Gastric Cancer}

Ricardo Oliveros Wilches, ${ }^{1}$ Helena Facundo, ${ }^{1}$ Carlos Bonilla González, ${ }^{2}$ Ricardo Sánchez Pedraza. ${ }^{3}$

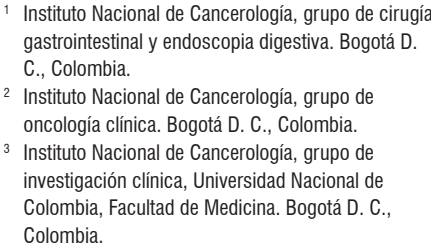
gastrointestinal y endoscopia digestiva. Bogotá D. C., Colombia.

2 Instituto Nacional de Cancerología, grupo de oncología clínica. Bogotá D. C., Colombia.

${ }^{3}$ Instituto Nacional de Cancerología, grupo de investigación clínica, Universidad Nacional de Colombia, Facultad de Medicina. Bogotá D. C. Colombia.

Fecha recibido: $08-05-17$ Fecha aceptado: 22-01-18

\begin{abstract}
Resumen
Se reporta el caso de una paciente de 47 años, con diagnóstico inicial de un tumor de Krukenberg por una lesión anexial de $10 \mathrm{~cm}$ de diámetro y una lesión corporal gástrica de $3 \mathrm{~cm}$. La biopsia mostró un adenocarcinoma pobremente diferenciado con células en anillo de sello. Una laparoscopia inicial mostró un índice de carcinomatosis peritoneal (ICP) de 24, por lo cual se le indica a la paciente quimioterapia con intención paliativa (cisplatino y capecitabina). Con mejoría clínica importante, respuesta adecuada y favorable a la quimioterapia, la paciente se remitió a una salpingo-ooforectomía bilateral. Continúa con quimioterapia evidenciándose mejoría de las imágenes tomográficas y una excelente respuesta clínica. Por este motivo, se decide en conjunto con la familia llevarla a cirugía. Se le practica una gastrectomía total con linfadenectomía D2 con intención curativa. La paciente recibe quimioterapia con capecitabina y cisplatino por 3 meses más, hasta cuando se evidencia compromiso ganglionar paraaórtico, por lo que es necesario reiniciar la quimioterapia con un nuevo esquema, entonces se le formula irinotecán. La paciente completa 22 meses desde el diagnóstico inicial, la condición clínica es muy buena y está asintomática.
\end{abstract}

\section{Palabras clave}

Cáncer gástrico, estadio IV, cirugía de conversión.

\section{Abstract}

We report the case of a 47-year-old patient initially diagnosed with a Krukenberg tumor, an adnexal lesion $10 \mathrm{~cm}$ in diameter and a $3 \mathrm{~cm}$ lesion in the gastric corpus. A biopsy showed a poorly differentiated adenocarcinoma with signet ring cells. Initial laparoscopy showed an index of peritoneal carcinomatosis of 24 which indicated chemotherapy with palliative intent (cisplatin and capecitabine). The patient improved significantly and underwent a total hysterectomy with salpingo-oophorectomy. Chemotherapy continued with excellent clinical response as evidenced in CT scans. Together with the patient's family, it was decided that she should undergo surgery. A total gastrectomy with D2 lymphadenectomy with curative intent was performed. The patient continued to receive capecitabine and cisplatin for three more months until para-aortic lymph node involvement was demonstrated and it became necessary to restart chemotherapy with a new scheme using iriniotecan. The patient has completed 22 months after the initial diagnosis in very good and clinical condition without symptoms.

Keywords

Stage IV gastric cancer, conversion surgery. 


\section{INTRODUCCIÓN}

El cáncer gástrico (CG) ha permanecido como una enfermedad prevalente en el mundo entero con un pronóstico muy pobre. En el año 2012 se diagnosticaron aproximadamente un millón de casos nuevos con CG y cerca de 700000 pacientes murieron a causa de esta enfermedad. El CG es el cuarto cáncer más común y la tercera causa de mortalidad por cáncer en el mundo (1). En Colombia, es la primera causa de mortalidad en los hombres y la tercera en las mujeres (2).

El CG generalmente se diagnostica en estados avanzados y menos del $30 \%$ sobrevive más de 5 años. Los pacientes con CG no resecables generalmente son candidatos a recibir quimioterapia sistémica antes de cualquier manejo quirúrgico a excepción de aquellos pacientes con indicación paliativa como la obstrucción o el sangrado (3). Sin embargo, también se cuenta con otras alternativas de paliación diferentes a la cirugía como la embolización por radiología para el sangrado y la colocación de stents por endoscopia para la obstrucción.

Varias combinaciones de nuevos regímenes de quimioterapia han permitido la conversión de un CG no resecable en un CG resecable. Esta cirugía adicional puede permitir una mayor supervivencia en pacientes seleccionados (4).

La cirugía de conversión es la resección radical realizada para lesiones no resecables, cánceres estadio IV y metastásicos que, luego de la administración de la quimioterapia con adecuada respuesta y regresión, se vuelven resecables técnica y oncológicamente (5).

El propósito de este trabajo es mostrar el caso clínico de una paciente con un CG estadio IV con varios factores no curativos, quien se remitió a cirugía en 2 oportunidades después de recibir un régimen combinado de quimioterapia. Se revisaron la evolución clínica, las tomografías y los hallazgos laparoscópicos y quirúrgicos con el resultado final de patolo- gía para evaluar el valor pronóstico de la cirugía de conversión. La última cirugía fue clasificada como R0 (resección completa sin tumor residual). La evolución de su control clínico se estableció con tomografías y marcadores tumorales (antígeno carcinoembrionario [ACE], antígeno carbohidrato [CA] 19-9) al menos cada 4 a 5 semanas.

\section{CASO CLÍNICO}

Paciente mujer de 47 años de edad, quien consulta en junio del 2015 por dolor abdominal en fosa ilíaca derecha de 4 meses de evolución. Se le practicó una ecografía abdominal en la que encontró una masa anexial derecha de $10 \mathrm{~cm}$ de diámetro. Una endoscopia digestiva alta mostró una lesión ulcerada de $3 \mathrm{~cm}$ de diámetro de bordes elevados localizada en la pared posterior del cuerpo gástrico, cuya patología mostró un adenocarcinoma pobremente diferenciado con células en anillo de sello, gastritis crónica no atrófica, sin metaplasia intestinal ni displasia y Helicobacter pylori negativo; la colonoscopia total fue normal; CA-125: $1396 \mathrm{U} /$ $\mathrm{mL}$; ACE $24,9 \mathrm{ng} / \mathrm{mL}$; creatinina $0,7 \mathrm{mg} / \mathrm{dL}$.

Una tomografía axial computarizada (TAC) de abdomen con contraste ( 24 de abril del 2015) demostró un leve aumento en el número de ganglios retroperitoneales. El útero estaba aumentado de tamaño con presencia de miomas y masa anexial derecha heterogénea de 110 x $79 x$ $71 \mathrm{~mm}$ de contornos lisos, bien definida y sólida; se encontraba una moderada cantidad de líquido libre en la cavidad; la pared gástrica corporal estaba engrosada; y el peritoneo era nodular (Figura 1).

La paciente fue valorada por gastroenterología y ginecología con diagnóstico de tumor de Krukenberg. Se decide llevar a laparoscopia el día 12 de junio del 2015 y se encontró líquido ascítico en cantidad de $400 \mathrm{~mL}$, múltiples implantes peritoneales en el peritoneo pélvico, cúpulas diafragmáticas, epiplón, masa ovárica derecha de $10 \mathrm{~cm}$ de diá-

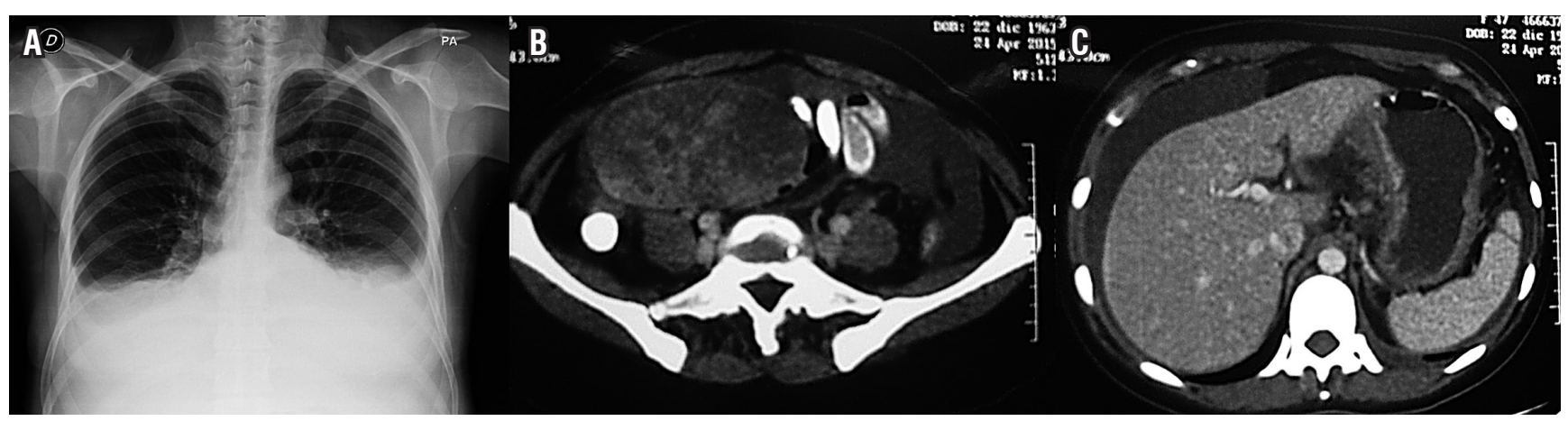

Figura 1. A. Radiografía de tórax, derrame plural. B. Tomografía con masa anexial derecha. C. Tomografía con líquido peritoneal y engrosamiento de paredes del estómago. 


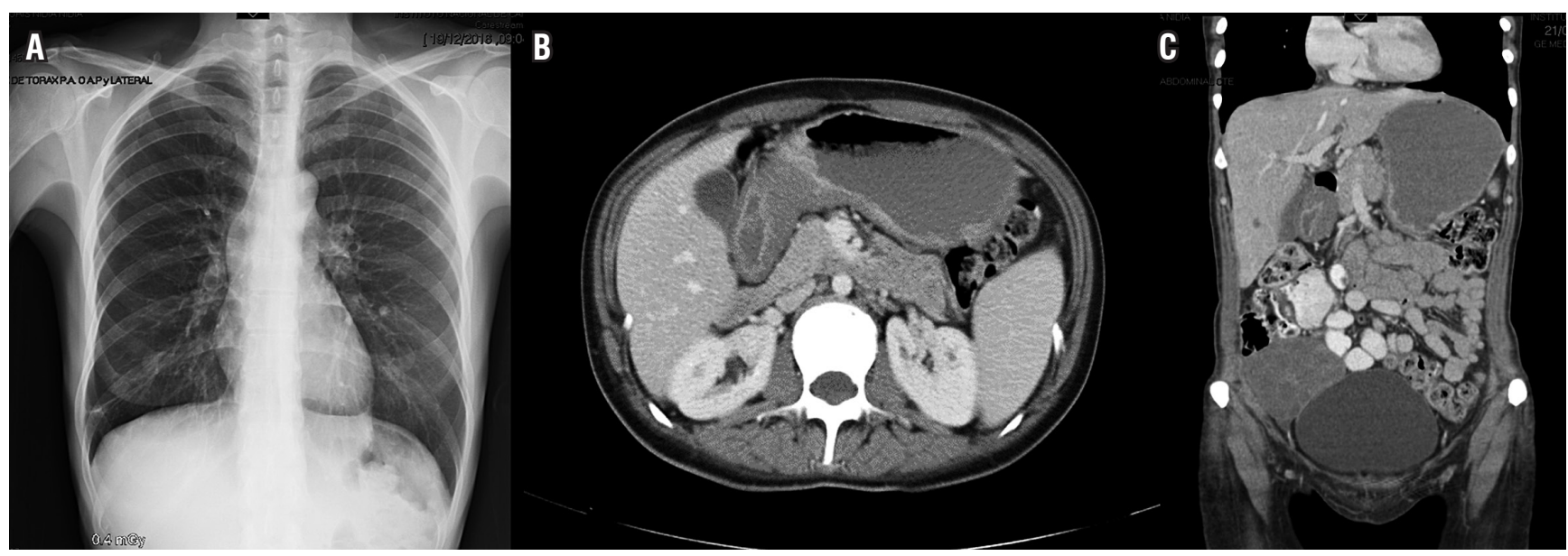

Figura 2. A. Radiografía de tórax normal. B. Tomografía con engrosamiento de las paredes del estómago- corporal. C. Masa anexial derecha.

metro y un tumor gástrico en la región corporal. El índice de carcinomatosis peritoneal (ICP) fue de 24. La citología de líquido ascítico resultó negativo para malignidad.

Es valorada por oncología. Se inicia la quimioterapia con capecitabina (1,5 g vía oral, cada 12 horas, del día 1 al 14) y cisplatino (125 mg intravenoso [IV], única dosis) desde el 7 de julio del 2015. Completó 8 ciclos. En sus controles periódicos se detectó un alivio clínico importante, recuperación de su estado funcional y nutricional, mejoría en las cifras de sus marcadores bioquímicos como el ACE, CA 19-9 y del CA-125. El seguimiento mostró desaparición de la ascitis y aumento de la masa ovárica. Por este motivo, se le propuso una nueva laparoscopia, que se realizó el 12 de noviembre del 2015 con el hallazgo de un ICP de 8 , sin implantes peritoneales en cúpulas diafragmáticas, estómago móvil sin adherencias y compromiso metastásico del ovario derecho. No hay líquido libre en cavidad abdominal (Figura 2).

Se presentó en junta quirúrgica con la propuesta de cirugía tipo Sugarbaker, dada la respuesta clínica al manejo con la quimioterapia sistémica. La junta concluyó que no hay beneficios con una cirugía citorreductora tipo Sugarbaker por el tipo histológico y el ICP. Se llevó a salpingo-ooforectomía bilateral, procedimiento que se realizó el día 13 de enero del 2016. Los hallazgos fueron tumores sólidos en ambos ovarios, el derecho de $15 \mathrm{~cm}$ y el izquierdo de $10 \mathrm{~cm}$, sin adherencias y sin implantes en el peritoneo; las cúpulas diafragmáticas estaban libres, había engrosamiento de las paredes gástricas; y el líquido ascítico fue de $500 \mathrm{~mL}$. La patología mostró ovario y trompa derecha positivos para adenocarcinoma mal diferenciado con componente de células en anillo de sello; invasión linfovascular positiva; y estudio de inmunohistoquímica positivo para queratina 19, queratina 7 y queratina 20 . El resultado fue negativo para human epidermal growth factor receptor 2 (HER2), CA-125, Wilms' tumor 1 (WT1), CDX2 y TF1. El ovario izquierdo resultó positivo para compromiso metastásico por adenocarcinoma con invasión linfovascular positiva.

La paciente continuó con quimioterapia, con capecitabina y cisplatino. El día 16 de mayo, por decisión conjunta con la familia, se llevó a cirugía: se realizó la gastrectomía total con disección ganglionar D2. El resultado de patología fue el producto de gastrectomía total, adenocarcinoma gástrico de tipo difuso con células en anillo de sello, lesión de 6 × 5 $\mathrm{cm}$, invasión tumoral hasta la grasa subserosa, invasión linfovascular y neural positivas, bordes proximal y distal libres de tumor, 5 de 17 ganglios positivos para compromiso tumoral, pT3pN2, epiplón negativo para tumor, rotulado diafragma izquierdo negativo para tumor, ganglios de la arteria hepática negativos para tumor, borde esofágico por aparte negativo para tumor y estado de HER2 negativo (Figura 3).

La paciente se remitió a oncología, donde se le ordenó 3 ciclos más de quimioterapia con cisplatino y capecitabina. En el último control en noviembre del 2016 resultó asintomática con marcadores tumorales elevados y un índice de Karnofsky del $100 \%$. Un control tomográfico evidenció un compromiso ganglionar paraaórtico. Se reinició un nuevo esquema de quimioterapia con irinotecán (250 mg IV, día 1 y día 15 ).

\section{DISCUSIÓN}

El tratamiento estándar del CG se ha establecido en varias guías. Para el estadio IV, la principal estrategia de manejo es la quimioterapia paliativa y la supervivencia media de estos pacientes es de solo 13 a 16 meses (6).

El CG estadio IV puede representar más del 30\% de los casos vistos al momento del diagnóstico (7); además, su 


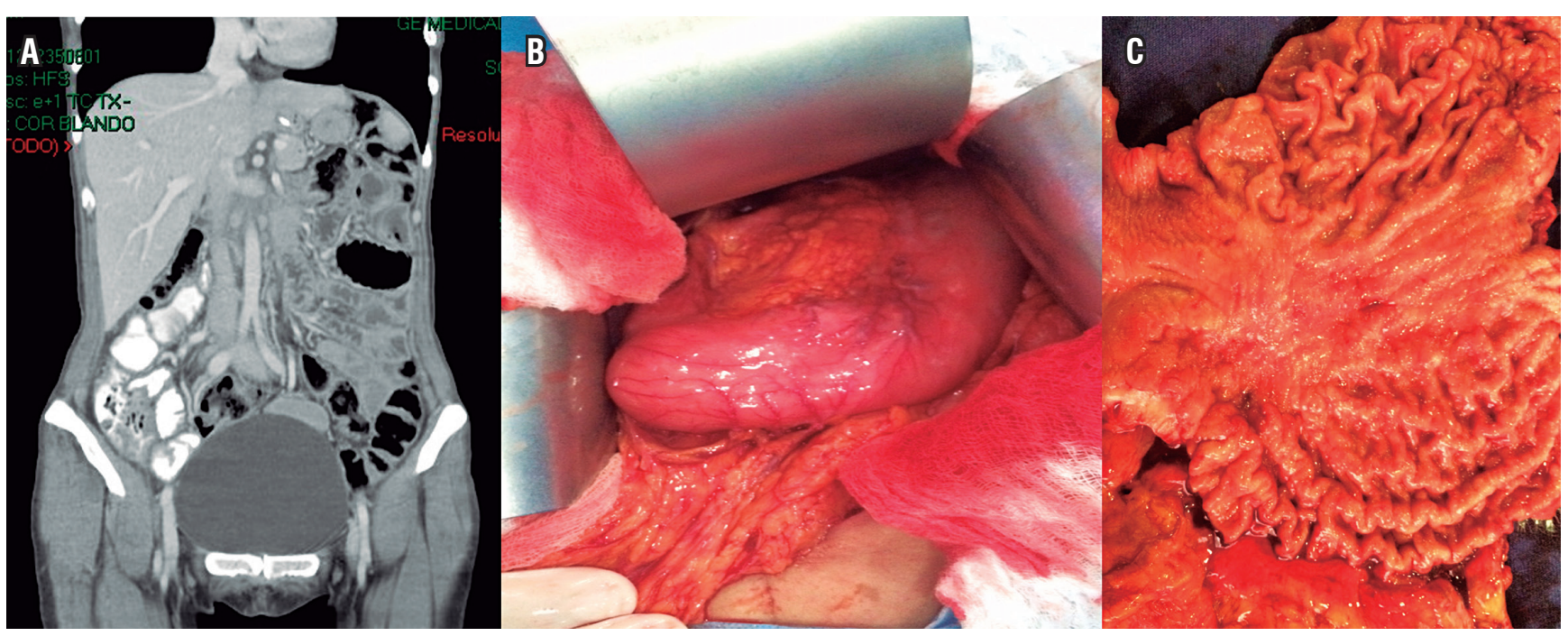

Figura 3. A. Tomografía de abdomen normal. B. Engrosamiento de las paredes de la región corporal gástrica. C. Pieza quirúrgica con lesión gástrica corporal.

presentación clínica es muy compleja y su espectro está representado por enfermedad metastásica que incluye lesiones hepáticas de diferente tamaño y número; metástasis en el peritoneo con localización supra e inframesocólica; metástasis ováricas, generalmente bilaterales y sólidas (tumor de Krukenberg); compromiso ganglionar a distancia y líquido ascítico positivo (8). Cuando se está con el paciente, en el ámbito clínico se utiliza el término de $C G$ no resecable, concepto usado muy comúnmente con implicaciones clínicas muy variables porque no representa la verdadera extensión de la enfermedad (5).

La paciente reportada representa un caso estadio IV, por tumor corporal gástrico con compromiso metastásico bilateral en los ovarios, compromiso peritoneal y presencia de líquido ascítico.

En este punto es importante diferenciar entre un CG no resecable por ser localmente avanzado; es decir, por estar adherido o unido a otros órganos o estructuras vasculares y un estadio IV, que implica la presencia de metástasis a distancia, como es el caso clínico reportado. Para ejemplificar mejor esta diferencia, se puede citar el caso de un estadio IV por adenopatías interaortocavas, que son ganglios no regionales y, por tanto, se consideran M1 (cáncer diseminado a otras partes del cuerpo). Técnicamente, un cirujano puede resecar estos ganglios además del tumor primario, pero se estaría tratando con cirugía inicial un estadio IV; es decir, hay irresecabilidad oncológica, aunque no técnica.

Las guías de la National Comprehensive Cancer Network (NCCN) para el CG estadio IV establecen el manejo paliativo y, de acuerdo con la condición funcional del paciente, tratamiento sistémico paliativo con quimioterapia o el mejor cuidado de soporte con intención sintomática. Los pacientes con índice de Karnofsky $>60 \%$ o un ECOG (Eastern Cooperative Oncology Group) <2 son candidatos para el manejo con quimioterapia paliativa o pueden ser incluidos en estudios clínicos controlados (9).

Cuando las guías de la European Society for Medical Oncology (ESMO) se refieren al CG no resecable o metastásico, indican la quimioterapia paliativa o el mejor cuidado de soporte si la condición funcional del paciente no es adecuada, pero también establecen una alternativa con indicación de cirugía para aquellos pacientes que han recibido quimioterapia paliativa. Dentro de esta guía se menciona: "sin embargo, un número pequeño de pacientes con enfermedad inicial localmente avanzada o no resecable pueden ser llevados a cirugía luego de una buena respuesta a la terapia sistémica." (10).

En las guías japonesas, la principal estrategia de manejo para el CG estadio IV es la quimioterapia paliativa (6). Para el CG metastásico y recurrente se han desarrollado nuevos regímenes de quimioterapia con el objetivo de mejorar la supervivencia. Actualmente, la combinación de 5-fluorouracilo (5-FU) o capecitabina y un análogo del platino (cisplatino u oxaliplatino) son los regímenes de primera línea más ampliamente utilizados para pacientes con CG no resecable o recurrente fuera del Japón (11). Se recomienda el docetaxel solamente después de falla de la quimioterapia de primera línea (11).

En los pacientes con cáncer colorrectal metastásico se han utilizado nuevos regímenes de quimioterapia y terapia blanco o terapia target con un incremento en la supervivencia de 6 a 30 meses. El abordaje quirúrgico de estas lesiones 
ulterior al manejo con quimioterapia ha jugado un papel crucial en la prolongación de la supervivencia (6). En el caso de los pacientes con CG metastásico o no resecables, este enfoque de manejo similar existe, pero es poco lo que se ha logrado en términos de supervivencia (12).

La introducción de nuevos agentes y el desarrollo de regímenes múltiples han permitido la conversión de un CG no resecable estadio IV en un CG resecable. Este tipo de cirugía referida como gastrectomía de conversión con intención curativa difiere de la gastrectomía paliativa (4). El estudio REGATTA demostró que la gastrectomía paliativa no está indicada a menos de que exista sangrado u obstrucción por la lesión primaria $(6,8)$.

La cirugía de conversión está indicada en pacientes con CG no resecable, quienes reciben quimioterapia por su estadio IV y después de una respuesta adecuada a la quimioterapia se llevan a cirugía con intención curativa. Esto ilustra las diferencias entre quimioterapia neoadyuvante y terapia de conversión (3).

La quimioterapia neoadyuvante se ha evaluado en pacientes con CG localmente avanzado sin metástasis. Este tipo de tratamiento tiene como propósito lograr una disminución en el estadio de la lesión primaria (downstage) para lograr mayores resecciones R0 y simultáneamente tratar las micrometástasis en sus formas más tempranas $(3,13)$. Este concepto es previo y diferente a la terapia de conversión.

La terapia de conversión para el CG estadio IV describe un concepto terapéutico diferente en el cual la estrategia de tratamiento se inicia con un nuevo esquema de quimioterapia que permite convertir tumores originalmente no resecables o marginalmente resecables en cirugías potencialmente curativas (R0) (6). Esta situación clínica está representada en el caso clínico reportado que, luego de corresponder a un estadio IV, la respuesta a la quimioterapia permite llevar a la paciente a una cirugía con intención curativa.

Dentro de las diferentes opciones para el manejo del CG estadio IV se mencionan la quimioterapia sistémica, la peritonectomía, la quimioterapia intraperitoneal y la quimioterapia intraperitoneal hipertérmica, que pueden mejorar el pronóstico de estos pacientes cuando su indicación lo permita (14).

El estadio IV del CG es una combinación de metástasis técnicamente resecables o no resecables, metástasis hematológicas, diseminación peritoneal y compromiso ganglionar extrarregional. Como existen diferentes consideraciones con el espectro de este estadio, se ha propuesto una nueva clasificación del estadio IV en varias categorías con el objetivo de clasificar la biológica del tumor y permitir un abordaje oncológico clínico y quirúrgico $(6,8)$. Esta nueva clasificación propone:

- Categoría 1: sin diseminación peritoneal, con metástasis potencialmente resecables: candidatos a quimioterapia neoadyuvante. Resección del tumor primario y de las metástasis logrando R0 $(6,8)$.

- Categoría 2: sin diseminación peritoneal con metástasis marginalmente resecables: quimioterapia intensiva, si es efectiva y cirugía con resección del tumor primario y de las metástasis logrando R0 $(6,8)$.

- Categoría 3: con diseminación peritoneal, incurable o no resecable: quimioterapia sistémica, quimioterapia intraperitoneal y terapia blanco. Cirugía citorreductora $(6,8)$.

- Categoría 4: con diseminación peritoneal y metástasis no resecables: quimioterapia, quimioterapia intraperitoneal y terapia blanco. Si esto no es efectivo, se lleva a quimioterapia paliativa $(6,8)$ (Figura 4).

Este concepto de terapia de conversión o cirugía adyuvante está dirigido a pacientes con categoría 2 , algunos con categoría 3 y muy pocos en categoría 4 . $(6,8)$.

El caso clínico reportado corresponde a un CG estadio IV, categoría 4, con muy buena respuesta a la quimioterapia y gastrectomía con intención curativa, evaluado en 2 oportunidades con laparoscopia para determinar la extensión de la enfermedad y respuesta al tratamiento.

Cuando la quimioterapia ha producido una respuesta adecuada y la cirugía curativa ha sido llevada a cabo, la cirugía de conversión está asociada con una supervivencia mayor en pacientes seleccionados (4).

Existen pocos reportes de cirugía de conversión en casos de CG. En el INC de Tokio, Nakajima fue el primero en reportar casos con cirugía de conversión. En 1977 reportó 30 casos con CG no resecables que recibieron 2 ciclos de quimioterapia con 5-FU, leucovorina, cisplatino y etopósido. Se obtuvo respuesta a la terapia en el 50\% de los pacientes y 19 se llevaron a cirugía. La cirugía curativa se logró en 9 pacientes (5).

Los pacientes tratados con quimioterapia más cirugía tuvieron una supervivencia significativamente mayor y tiempo libre de progresión a los 5 años, en comparación con los pacientes tratados solo con quimioterapia. Yoshida utilizó el término de cirugía adyuvante, en la cual la cirugía se realiza después de una respuesta adecuada a la quimioterapia (6).

Einama y colaboradores reportaron 10 pacientes entre el 2009 y 2015 con CG estadio IV llevados a cirugía radical luego de haber recibido quimioterapia. El tiempo promedio entre el inicio de la quimioterapia y el momento de la cirugía fue de 210 días. Todos los pacientes sobrevivieron más de un año y 5 estaban sin recurrencia al momento de su publicación. El tiempo promedio de supervivencia fue de 871 días luego del diagnóstico. Por esta razón, la resección curativa después de la quimioterapia que es efectiva está asociada con un mejor pronóstico. Igualmente, se demostró que los pacientes llevados a cirugía de conversión 


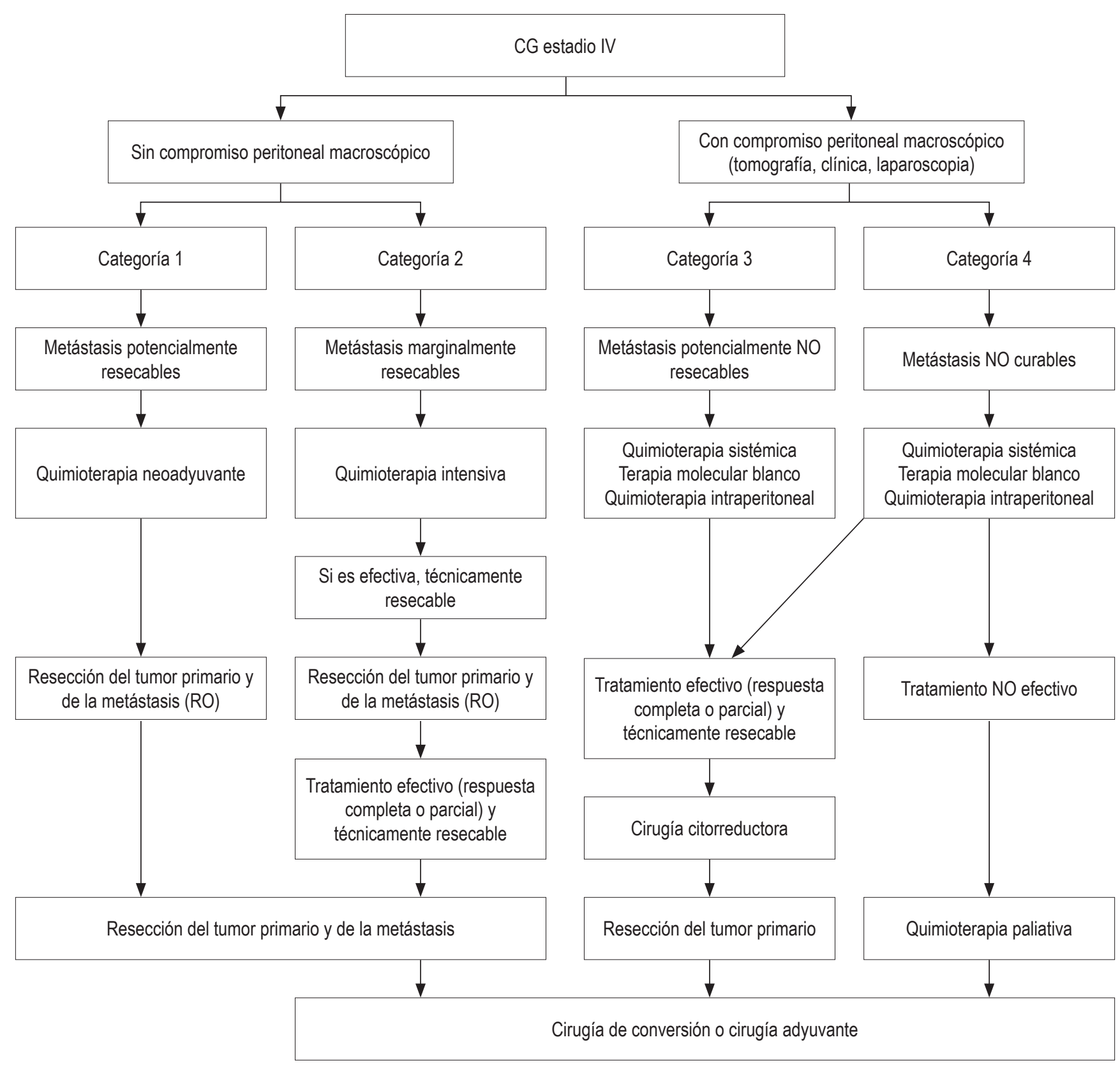

Figura 4. Tratamiento para las nuevas categorías del CG Estadio IV (6). Modificado de: Yoshida K et al. Gastric Cancer. 2016;19(2):329-38.

tienen una mejor sobrevida comparados con esos que solo recibieron quimioterapia (15).

En conclusión, el tema de la cirugía o terapia de conversión conlleva una serie de preguntas que necesitan respuestas (8):

1. ¿Cuál es la indicación? Las indicaciones para la terapia de conversión incluyen pacientes con categoría 2, algunos pacientes con categoría 3 y muy pocos pacientes con categoría 4 (8).
2. ¿Cuál es el mejor régimen de quimioterapia? Los regímenes de quimioterapia son la combinación del S1, 5-FU o capecitabina con un platino (cisplatino, oxiplatino o carboplatino) o docetaxel (12).

3. ¿Cuál es el mejor momento de la cirugía? El mejor momento para la cirugía es aquel en el que el tumor logra la mayor respuesta a la quimioterapia, casi de forma similar a la manera como se evalúa la respuesta 
en los pacientes que reciben terapia para el tumor del estroma gastrointestinal (GIST). Generalmente, la respuesta completa o parcial se logra luego de 4 a 6 ciclos de quimioterapia combinada. Algunos autores han reportado beneficio con solo 2 ciclos (11).

4. ¿Es necesario dar quimioterapia luego de la cirugía? La quimioterapia debe continuarse después de la cirugía hasta que el tumor adquiera resistencia o aparezcan efectos adversos por el tratamiento instaurado (6).

El valor clínico de la terapia multimodal incluyendo la quimioterapia y la cirugía de conversión para el CG estadio IV permanece con mucha controversia porque se trata de pacientes con lesiones inicialmente no resecables por la presencia de una enfermedad avanzada con diseminación sistémica (4), que incluye un comportamiento biológico variable y un espectro de condiciones clínicas. Sin embargo, el comportamiento biológico del tumor frente al manejo con quimioterapia puede definir un grupo de pacientes con una excelente respuesta que permite convertir la intención del tratamiento de paliación a curación.

\section{REFERENCIAS}

1. den Hoed CM, Kuipers EJ. Gastric Cancer: How Can We Reduce the Incidence of this Disease? Curr Gastroenterol Rep. 2016;18(7):34. https://doi.org/10.1007/s11894016-0506-0.

2. International Agency for Research of Cancer, World Health Organization. GLOBOCAN 2012: estimated cancer incidence, mortality and prevalence worldwide in 2012. IARC [internet] 2012. Disponible en: http://globocan.iarc.fr/ default.aspx.

3. Fukuchi M, Ishiguro T, Ogata $\mathrm{K}$, et al. Prognostic role of conversion surgery for unresectable gastric cancer. Ann Surg Oncol. 2015;22(11):3618-24. https://doi.org/10.1245/ s10434-015-4422-6.

4. Kinoshita J, Fushida S, Tsukada T, et al. Efficacy of conversion gastrectomy following docetaxel, cisplatin, and S-1 therapy in potentially resectable stage IV gastric cancer. Eur J Surg Oncol. 2015;41(10):1354-60. https://doi. org/10.1016/j.ejso.2015.04.021.
5. Terashima M. Conversion therapy for gastric cancer: who can make conversion as successful as Goromaru? Gastric Cancer. 2016;19(3):685-6. https://doi.org/10.1007/ s10120-016-0609-1.

6. Yoshida K, Yamaguchi K, Okumura N, et al. Is conversion therapy possible in stage IV gastric cancer: the proposal of new biological categories of classification. Gastric Cancer. 2016;19(2):329-38. https://doi.org/10.1007/s10120-0150575-z.

7. Oliveros R, Navarrera LF. Diagnóstico, estadificación y tratamiento del cáncer gástrico en Colombia desde 2004 a 2008 (REGATE-Colombia). Rev Col Gastroenterol. 2012; 27(4):269-74.

8. Yamaguchi K, Yoshida K, Tanaka Y, et al. Conversion therapy for stage IV gastric cancer-the present and future. Transl Gastroenterol Hepatol. 2016;1:50. https://doi. org/10.21037/tgh.2016.05.12.

9. Ajani JA, D’Amico TA, Almhanna K, et al. Gastric Cancer, Version 3.2016, NCCN Clinical Practice Guidelines in Oncology. J Natl Compr Canc Netw. 2016;14(10):1286-312. https://doi.org/10.6004/jnccn.2016.0137.

10. Smyth EC, Verheij M, Allum W, et al. Gastric cancer: ESMO Clinical Practice Guidelines for diagnosis, treatment and follow-up. Ann Oncol. 2016;27(suppl 5):v38-v49. https:// doi.org/10.1093/annonc/mdw350.

11. Takashima A, Yamada Y, Nakajima TE, et al. Standard firstline chemotherapy for metastatic gastric cancer in Japan has met the global standard: evidence from recent phase III trials. Gastrointest Cancer Res. 2009;3(6):239-44.

12. Wang S, Yuan L. Predictive biomarkers for targeted and cytotoxic agents in gastric cancer for personalized medicine. Biosci Trends. 2016;10(3):171-80. https://doi. org/10.5582/bst.2016.01078.

13. Sakamoto J. Neoadjuvant chemotherapy: a standard treatment for locally advanced gastric cancer in the near future? Gastric Cancer. 2003;6(3):131-3. https://doi. org/10.1007/s10120-003-0254-3.

14. KimSW.The result of conversion surgery in gastric cancer patients with peritoneal seeding. J Gastric Cancer. 2014;14(4):266-70. https://doi.org/10.5230/jgc.2014.14.4.266.

15. Einama T, Abe H, Shichi S, et al. Long-term survival and prognosis associated with conversion surgery in patients with metastatic gastric cancer. Mol Clin Oncol. 2017;6(2):163166. https://doi.org/10.3892/mco.2017.1128. 